\title{
Musculoskeletal ultrasound as a biomarker of remission - results from a one-year prospective study in patients with rheumatoid arthritis
}

\author{
Tanya Sapundzhieva, Rositsa Karalilova, Anastas Batalov
}

Medical University of Plovdiv, Medical Faculty, Department of Propaedeutic of Internal Diseases, Rheumatology Clinic, University Hospital 'Kaspela', Plovdiv, Bulgaria

\begin{abstract}
Aims: To assess the role of musculoskeletal ultrasound (MSUS) as a biomarker of remission and to compare the rates of clinical and imaging remission in patients with rheumatoid arthritis (RA) on different types of treatment. Material and methods: One hundred and forty-one patients underwent physical and ultrasound examination at 5 visits (at baseline and after 1, 3, 6 and 12 months). Patients were divided into two groups according to the type of treatment, which involved synthetic (sDMARDs) and biologic (bDMARDs) disease-modifying antirheumatic drugs. Ultrasound assessment of the wrist, second and third metacarpophalangeal, second and third proximal interphalangeal joints, and the second and fifth metatarsophalangeal joints was performed on gray scale ultrasound (GSUS) and on power Doppler ultrasound (PDUS) (German US7-score). The rate of imaging and clinical remission (DAS28, SDAI, CDAI, and Boolean) was established. The percentage of patients in clinical remission with persistent PD signal was assessed. Results: In the sDMARDs group at month twelve, $43.6 \%$ of the patients achieved DAS2 8 remission, 5.1\% - SDAI, 3.8\% - CDAI, and 3.8\% - Boolean remission. In the bDMARDs group $49.2 \%$ achieved DAS2 8 remission, $6.3 \%$ - SDAI, $4.8 \%$ - CDAI, and 4.8\% - Boolean remission. Irrespective of which clinical index was applied, all patients in clinical remission had persistent synovial hypertrophy on GSUS. Synovial PD signal (PDUS score $\geq 1$ ) was detected in $77 \%$ and $71 \%$ of patients in DAS28 remission in the sDMARDs and bDMARDs group, respectively. Patients in SDAI, CDAI and Boolean remission in both treatment groups did not have a positive PD signal. Conclusions: There is persistence of synovitis both in patients on sDMARDs and bDMARDs in DAS28 clinical remission. This fact points to a discordance between DAS28 clinical remission and the imaging remission assessed by MSUS irrespective of the type of treatment. MSUS may be a feasible imaging method for the assessment of residual inflammation in daily rheumatology practice.
\end{abstract}

Keywords: ultrasound; remission; rheumatoid arthritis; biomarker

\section{Introduction}

Musculoskeletal ultrasound (MSUS) is included in the European League Against Rheumatism (EULAR) recommendations as a valuable imaging tool in patients with rheumatoid arthritis (RA) [1]. In an ideal case, remission is defined as an absence of disease symptoms

Received 03.06.2018 Accepted 17.09.2018

Med Ultrason

2018, Vol. 20, No 4, 453-460

Corresponding author: Tanya Sapundzhieva

Rheumatology Clinic, University Hospital

'Kaspela', block 2

64 Sofia street, 4002 Plovdiv, Bulgaria

Phone: +359 883504605

E-mail address: taniasapundjieva@abv.bg and development of structural progression and functional deficit over time [2]. However, evidence exists that some patients with RA experience radiographic progression despite being in clinical remission [3]. This may be explained by the persistence of subclinical joint inflammation which can only be detected through sensitive imaging techniques such as MSUS and magnetic resonance imaging [4-6]. MSUS has been proved to be more sensitive than physical examination for the detection of synovitis [7]. Evidence exists that the presence of a power Doppler (PD) signal in RA patients in clinical remission predicts structural progression and a recent relapse [4,8-10].

Studies have shown that clinical remission established on the basis of different indices (Disease Activity Score for 28 joints (DAS28), DAS $28 \leq 2.6$ [11]; Sim- 
plified Disease Activity Index (SDAI), SDAI $\leq 3.3$ [12]; Clinical Disease Activity Index (CDAI), CDAI $\leq 2.8$ [13]; ACR/EULAR definition of remission, 2011 [2]) does not entirely correspond to imaging remission. In order to improve disease outcomes US examination of the joints in patients in clinical remission may be used to guide therapeutic decisions, for example drug tapering [14-17].

Prior to use MSUS for the assessment of remission, the following questions should be addressed: 1) How many and which joints should be examined?; 2) Which scan, dorsal and/or volar, of hand joints should be used?; 3) What should be the position of the joints during the exam?; and 4) Which definition and grading scales for synovitis should be used? [18]. There is evidence that shows that the results of scoring a reduced number of joints correlate to a great extent with those involving the assessment of 78 joints [19]. There are accepted US definitions of synovial hypertrophy, effusion and tenosynovitis [20]. Regarding the most appropriate scan, there is evidence that synovitis is more frequently detected through a palmar scan of metacarpophalangeal (MCP) and proximal interphalangeal (PIP) joints in their proximal part than through a dorsal scan $[21,22]$. Different US scores exist for the assessment of disease activity and of treatment response. The German US7-score has been demonstrated to reflect disease activity and to be suitable for monitoring therapy in daily rheumatology practice $[23,24]$.

There are different definitions of US remission. Some authors define it as an absence of joints with PD signal $[9,25-27]$. Others accept a more stringent definition, which requires the absence of synovitis both on a gray scale (GS) and PDUS [28]. Van der Ven et al (2017) accept a minimal amount of synovitis on GS and define US remission as a gray scale grade of synovitis $\leq 1$ and power Doppler grade of synovitis $=0$ for each scanned joint [29]. Some authors accept a minimal residual PD signal (total PD activity score $\leq 1$ ) [30].

The primary aim of our study was to assess the role of US examination (US7 score) as a biomarker for remission and to compare the rates of clinical and imaging remission in a cohort of patients with RA on different types of treatment followed for a period of one year. Secondary goals were to find which definition of clinical remission best correlates with the presence of US remission, i.e. which is the clinical definition characterized by the lowest rate of PD positive synovitis in both treatment groups.

\section{Material and methods}

\section{Patients}

One hundred and forty-one patients with RA were consecutively enrolled in this prospective study between
April 2016 and January 2018. Inclusion criteria were: diagnosis of RA according to ACR/EULAR 2010 classification criteria [31] and moderate or high disease activity according to DAS28(CRP). Patients were divided into two groups as standard of care according to the treating rheumatologist: Group 1 included patients treated with synthetic disease-modifying antirheumatic drugs (sDMARDs) and Group 2 was comprised of patients on biologic DMARDs (bDMARDs). After enrollment in the study, therapy was either initiated (in patients with newly diagnosed RA) or escalated according to the treat-to-target strategy. The rate of clinical and imaging remission was recorded. All patients underwent physical and ultrasound examination at 5 visits - at baseline (Visit $0-\mathrm{V} 0$ ) and after 1 (V1), 3 (V2), 6 (V3) and 12 (V4) months. The study was approved by the Ethical Committee of the Medical University of Plovdiv. Informed consent was obtained from all subjects.

\section{Clinical assessment}

Twenty-eight joints were evaluated by the same assessor for swelling and/or tenderness: bilaterally PIP and MCP joints, wrist, elbow, shoulder and knee joints. Pain intensity was measured on a visual-analogue scale (VAS) from $0-10 \mathrm{~cm}$. The assessor was blinded to the patients' treatment group/type. For assessment the disease activity three indices were calculated-DAS28, SDAI and CDAI.

\section{Laboratory parameters}

C-reactive protein (CRP) level (normal value $\leq 6.0$ $\mathrm{mg} / \mathrm{l})$ was obtained at baseline, V2 and V4. IgM-Rheumatoid factor (IgM-RF: normal value $<20 \mathrm{U} / \mathrm{l})$ and anti-citrullinated protein antibodies (ACPA: normal value $<20 \mathrm{U} / \mathrm{l})$ were assessed at baseline.

\section{Ultrasound assessment}

US assessment was conducted by an assessor who was blinded to the patients' clinical data and type of treatment. MSUS of wrist, hands and forefoot was performed using MyLab 7, Esaote, Italy machine equipped with a multi-frequency linear probe (10-18 MHz). GSUS frequency was $12-18 \mathrm{MHz}$ depending on the examined joint and GSUS gain was estimated based on joint regions and patients, yielding an average of 50\%. Settings for PDUS were as follows: frequency $9.1 \mathrm{MHz}$; pulse repetition frequency $500-750 \mathrm{~Hz}$; PDUS gain in relation to joint regions and patients, amounting to $50 \%$ average; low wall filter We examined by GSUS and PDUS 7 joints of the clinically dominant hand/foot, affected more by swelling or tenderness, by using the German US7-score (wrist, second and third MCP and PIP, second and fifth metatarsophalangeal (MTP) joints [23,24]. Several parameters were assessed according to the Outcome Measures in Rheumatology (OMERACT) definitions of pathology, including presence of synovitis, tenosynovitis/paratenon- 
itis and erosions [20]. The wrist joint was assessed for synovitis and tenosynovitis on dorsal, palmar and ulnar scan. Palmar scan was used to assess MCP2 and MCP3 for synovitis and tenosynovitis; and dorsal scan for paratenonitis. Erosions were assessed on the dorsal, palmar and for MCP2 also on the radial scan. PIP2 and PIP3 were assessed for synovitis on the palmar scan and for erosions on the dorsal and palmar scan. MTP2 and MTP5 were assessed for synovitis on the dorsal scan and for erosions on the dorsal and plantar scan (for MTP2) and on the dorsal, plantar and lateral scan (for MTP5). Synovitis on GSUS was scored on a semi-quantitative scale (0 to 3) [21]. Tenosynovitis/paratenonitis and erosions were documented as present (1) or absent (0).

PDUS was used for grading of synovitis and tenosynovitis/paratenonitis on the dorsal and palmar scan for each joint. PDUS of MTP joints was performed only on dorsal scan. Synovitis and tenosynovitis/paratenonitis on PDUS were scored on a semi-quantitative scale (grade $0-3)[32,33]$.

The scoring range was $0-27$ for GSUS synovitis, $0-7$ for GSUS tenosynovitis/paratenonitis, 0-39 for PDUS synovitis, and 0-21 for PDUS tenosynovitis/paratenonitis. US7-score was calculated as the sum of the synovitis score and tenosynovitis/paratenonitis score on GSUS and of the synovitis and tenosynovitis scores on PDUS. The sonographic examination of each patient took approximately 10-20 minutes, including documentation. US remission was defined as an absence of joints with PD signal, i.e. total PDUS score $=0$ [25].

\section{Statistical analysis}

The data was analyzed with the Statistical Package for the Social Sciences (SPSS), Version 24 [34]. Descrip- tive statistics included mean values \pm standard deviation (SD) for continuously measured and normally distributed variables, and frequencies and percentages for dichotomous variables. The continuous demographic (age and disease duration), clinical (DAS28, SDAI, CDAI, CRP, HAQ, VAS) and sonographic (scores for GS synovitis, PD synovitis, GS tenosynovitis, PD tenosynovitis, erosion score (ErS), GSUS score, PDUS score, US7 score) variables at baseline were checked for normality with the Kolmogorov-Smirnov test which showed that in both the sDMARDs and bDMARDs group the assumption of normality was in place, $(p>0.05)$. The baseline demographic, clinical and sonographic parameters in the treatment groups (sDMARDs and bDMARds) were compared through an independent samples t-test. The proportions of baseline dichotomous data (gender, ACPA positive and IgM-RF positive) and the observed clinical and sonographic remission in the course of the treatment between the two groups were examined through the chisquare test. Statistical significance was considered at Type I error rate alpha $\leq 0.5$.

\section{Results}

\section{Characteristics of patients}

One hundred and forty-one RA patients (79.0\% women) of mean $\pm \mathrm{SD}$ age of $58.90 \pm 11.04$ years and mean $\pm \mathrm{SD}$ disease duration of $87.62 \pm 97$ months were enrolled. At inclusion, 90 patients $(64.0 \%)$ were IgM-RF positive and 120 patients $(81.0 \%)$ were ACPA positive. Seventy-eight patients $(55.3 \%)$ were treated with sDMARDs and 63 (44.7\%) with bDMARDs. In the sDMARDs group 69 patients $(88.5 \%)$ were treated with conventional sDMARDs

Table I. Patients' demographic and clinical data at baseline

\begin{tabular}{|c|c|c|c|c|c|}
\hline \multirow{2}{*}{ Variables } & & \multirow{2}{*}{$\begin{array}{l}\text { Total } \\
(\mathrm{N}=141)\end{array}$} & \multicolumn{3}{|l|}{ GROUP } \\
\hline & & & SDMARDs $(\mathrm{N}=78)$ & bDMARDs $(\mathrm{N}=63)$ & $\mathbf{p}$ \\
\hline Age & & $58.90 \pm 11.04$ & $59.47 \pm 11.37$ & $58.20 \pm 10.68$ & 0.500 \\
\hline \multirow[t]{2}{*}{ Gender } & Female & $111(79)$ & $65(83)$ & $46(73)$ & \\
\hline & Male & $30(21)$ & $13(17)$ & $17(27)$ & 0.137 \\
\hline \multirow[t]{2}{*}{ Disease duration } & Months & $87.62 \pm 97$ & $73.5 \pm 101$ & $105.20 \pm 89.26$ & 0.053 \\
\hline & Newly diagnosed & $23(16)$ & $22(28.2)$ & $1(1.6)$ & 0.0001 \\
\hline ACPA positive & & $120(81)$ & $59(75.6)$ & $61(96.8)$ & 0.0001 \\
\hline IgM-RF positive & & $90(64)$ & $47(60)$ & $43(68.3)$ & 0.30 \\
\hline DAS28 & & $5.43 \pm 0.92$ & $5.37 \pm 0.90$ & $5.49 \pm 0.93$ & 0.455 \\
\hline SDAI & & $33.80 \pm 11.97$ & $32.90 \pm 11.58$ & $34.71 \pm 12.37$ & 0.372 \\
\hline CDAI & & $31.02 \pm 11.35$ & $30.18 \pm 11.26$ & $31.87 \pm 11.44$ & 0.381 \\
\hline CRP (mg/l) & & $27.47 \pm 26.50$ & $27.23 \pm 19.62$ & $28.32 \pm 33.50$ & 0.811 \\
\hline HAQ & & $1.54 \pm 0.63$ & $1.49 \pm 0.66$ & $1.72 \pm 0.60$ & 0.035 \\
\hline VAS $(0-100)$ & & $74.18 \pm 12.75$ & $72.95 \pm 11.96$ & $75.40 \pm 13.53$ & 0.257 \\
\hline
\end{tabular}

The results are expressed as number (\%) or mean \pm SD; N- number of patients; DAS28 - Disease Activity Score for 28 joints; SDAI - Simplified Disease Activity Index; CDAI - Clinical Disease Activity Index; CRP - C-reactive protein; IgM-RF - IgM-Rheumatoid factor; ACPA - anti-citrullinated protein antibodies; HAQ - Health Assessment Questionnaire; VAS -visual-analogue scale. 
Table II. Ultrasonographic data at baseline

\begin{tabular}{lllll}
\hline US scores & Total & \multicolumn{3}{c}{ GROUP } \\
\cline { 2 - 5 } & $(\mathbf{N}=\mathbf{1 4 1})$ & sDMARDs (N= 78) & bDMARDs (N= 63) & $\mathbf{p}$ \\
\hline GS synovitis & $12.47 \pm 3.55$ & $12.33 \pm 3.69$ & $12.62 \pm 3.39$ & 0.642 \\
PD synovitis & $6.8 \pm 3.85$ & $6.9 \pm 4.24$ & $6.7 \pm 3.47$ & 0.372 \\
GS tenosynovitis & $1.55 \pm 1.15$ & $1.52 \pm 1.23$ & $1.59 \pm 1.04$ & 0.704 \\
PD tenosynovitis & $0.68 \pm 1.34$ & $0.67 \pm 1.32$ & $0.70 \pm 1.37$ & 0.890 \\
ErS & $4.18 \pm 3.87$ & $3.12 \pm 3.70$ & $5.24 \pm 4.05$ & $\mathbf{0 . 0 0 2}$ \\
GSUS & $14.06 \pm 4.07$ & $13.9 \pm 4.15$ & $14.2 \pm 4.00$ & 0.711 \\
PDUS & $7.44 \pm 4.62$ & $7.5 \pm 5.10$ & $7.4 \pm 4.14$ & 0.980 \\
US7 & $21.46 \pm 8.03$ & $21.38 \pm 8.63$ & $21.54 \pm 7.43$ & 0.910 \\
\hline
\end{tabular}

The results are expressed as mean $\pm \mathrm{SD}$; ErS - Erosion score; US - ultrasonography: GS - gray scale; PD - power Doppler; N - number of patients

Table III. Clinical remission rates after 12 months and ultrasonographic remission rates after 3, 6 and 12 months

\begin{tabular}{lllll}
\hline Clinical indices & \multicolumn{4}{c}{ Clinical remission (after 12 months) } \\
\cline { 2 - 5 } & sDMARDs & bDMARDs & p \\
\hline DAS28 & $34(43.6)$ & $31(49.2)$ & 0.437 & 0.51 \\
SDAI & $4(5.1)$ & $4(6.3)$ & 0.094 & 0.76 \\
CDAI & $3(3.8)$ & $3(4.8)$ & 0.085 & 0.77 \\
Boolean & $3(3.8)$ & $3(4.8)$ & 0.085 & 0.77 \\
\cline { 2 - 5 } & & & PDUS remission & 0.77 \\
Month 3 & $3(3.8)$ & $3(4.8)$ & 0.085 & 0.77 \\
Month 6 & $3(3.8)$ & $9(4.8)$ & 0.085 & 0.62 \\
Month 12 & $9(11.5)$ & $9(14.3)$ & 0.244 & \\
\hline
\end{tabular}

The results are expressed as number (\%); DAS28 - Disease Activity Score for 28 joint; SDAI - Simplified Disease Activity Index; CDAI - Clinical Disease Activity Index; US - ultrasonography: GS - gray scale; PD - power Doppler; N - number of patients

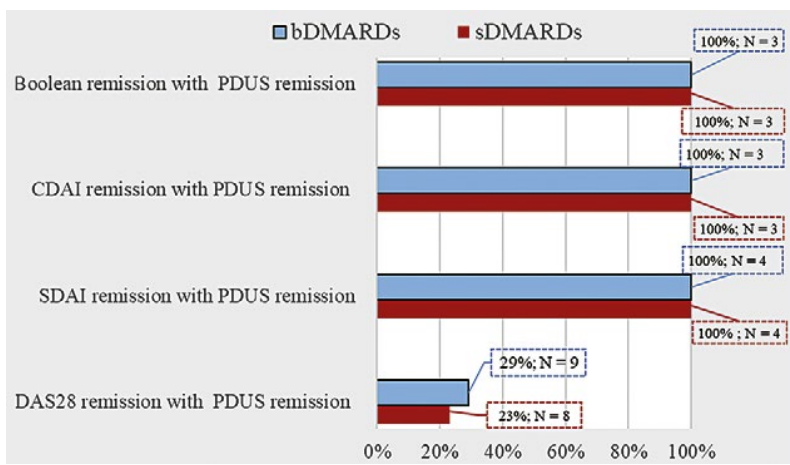

Fig 1. Triangulation of clinical and ultrasonographic remission in the sDMARDs and bDMARDs groups.

(csDMARDs) and 9 patients (11.50\%) with targeted sDMARDs. In the bDMARDs group 47 patients $(75 \%)$ were on combination therapy - csDMARDS and bDMARDs and 16 patients $(25 \%)$ were on bDMARDs monotherapy.

The two treatment groups did not differ significantly in the clinical indices for disease activity, CRP level and VAS pain level (table I).

The difference in the baseline US scores of the two treatment groups was not statistically significant, except

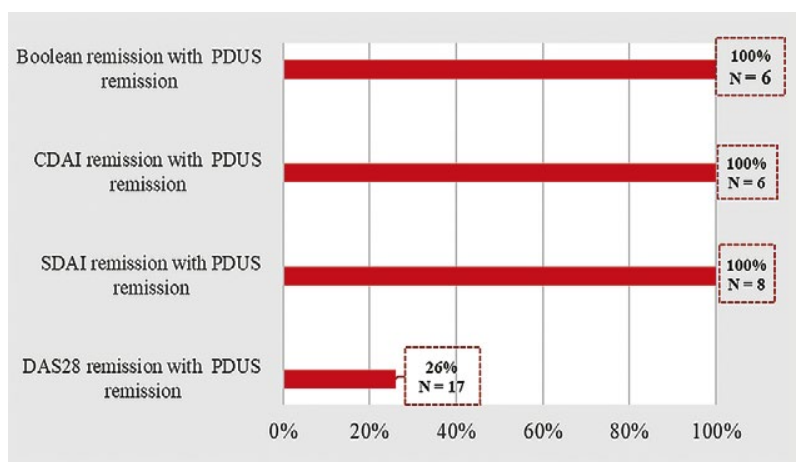

Fig 2. Rates of clinical remission with ultrasonographic remission in all rheumatoid arthritis patients.

for the erosion score, which was significantly higher in the bDMARDs group, $\mathrm{p}=0.002$ (table II).

Clinical and sonographic remission after one year

The clinical and sonographic remission rates after 12-month treatment are summarized in table III. The highest rate of remission was observed in relation to DAS28. At month 12 none of the patients had achieved Gray Scale score of 0 ; hence, the results in table III summarize US remission according to PDUS (PDUS $=0$ ). 
Triangulation of the results of the clinical and PDUS remission is illustrated in figure 1. DAS28 remission with PD remission was present in $8(23 \%)$ of the sDMARDs patients and in $9(29 \%)$ of those in the bDMARDs group. Remission according to the rest of the clinical indices, SDAI, CDAI and Boolean remission, showed 100\% overlap with PDUS remission in both patients' groups.

In the sDMARDs group, PD remission was present in $8(23 \%)$ patients in DAS28 remission and in all patients in SDAI, CDAI and Boolean remission. Synovial PD signal (PDUS score $\geq 1$ ) was detected in $26(77 \%)$ of patients in DAS28 remission. In the bDMARDs group, PD remission (PDUS score $=0$ ) was present in $9(29 \%)$ of patients in DAS28 remission, and in all patients in SDAI, CDAI and Boolean remission. Synovial PD signal (PDUS score $\geq 1$ ) was detected in $22(71 \%)$ of patients in DAS28 remission.

Altogether, 65 RA patients (46\%) achieved DAS28 remission and PDUS remission was present in $17(26 \%)$ of these patients (fig 2).

\section{Discussion}

The achievement of true remission became a more realistic goal after the introduction of biologic treatment and "treat-to-target" strategy $[35,36]$. Evidence exists that all patients in clinical remission, irrespective of the used definition, may experience progression of the radiographic damage [37,38]. Nevertheless, research shows that stricter definitions are associated with a reduced likelihood of structural progression [37]. US examination as a sensitive imaging technique for the detection of synovitis may be needed to determine imaging remission [39-41]. This is important as US-detected synovitis in clinical remission is a predictor for a relapse and loss of remission $[9,10,42]$.

A biomarker is an objectively measured indicator of the status of a biologic process or a disease. It defines various aspects of pathogenesis, disease activity, therapy response or a disease outcome [43]. The association of PDUS with histological findings supports the current opinion that PDUS is a biomarker of treatment response and reflects both clinical and histological markers of disease activity in patients with RA $[44,45]$.

The aim of our study was to assess the role of the US7 score as a biomarker of remission and to compare the rates of clinical and imaging remission in a cohort of patients with RA receiving different types of treatment. Previous studies have shown that more patients achieve DAS28 remission as compared to CDAI, SDAI and ACR/ EULAR remission $[46,47]$ but at the same time more patients in DAS28 remission have persistent PD positive synovitis in comparison to patients in SDAI, CDAI or ACR/EULAR remission $[39,46,48,49]$. The results of our study are in agreement with the results of these studies. We decided to define imaging remission only on the basis of the PDUS score, ignoring GS changes because their significance in later RA remains uncertain [50]. We found that the rate of US remission at month 12 was higher for the bDMARDs group as compared to the sDMARDs group although the difference did not reach the significance level. Other authors have also found that the rate of imaging remission does not depend on the type of treatment (conventional or biologic) [51].

At month twelve, all patients had persistent synovial hypertrophy on GSUS (GSUS score $\geq 1$ ) and 48 patients (74\%) patients in DAS28 remission had a persistent PD signal. All patients in CDAI, SDAI and Boolean remission had a negative PD signal (PDUS score $=0$ ). An important conclusion from our study is that there is an agreement between US remission and SDAI, CDAI and Boolean remission and a discordance between US and DAS28 remission both in patients on sDMARDs and on bDMARDs. Our opinion is that the SDAI, CDAI and Boolean definitions of remission are stricter than the DAS28 definition.

The problem of discordance between clinical and US remission was largely studied in the last years. Balsa et al have demonstrated that in relation to imaging remission there were no differences between DAS28 and ACR criteria, but SDAI better correlated with US remission [25]. Differences between DAS28 and SDAI were also demonstrated by Naredo et al [48]. Peluso et al showed a high proportion of patients with persistent synovitis using DAS criteria compared to the ACR criteria [9], while in a recent study Olmez et al demonstrated that CDAI was superior to other indices to assess remission [49]. Contrarily, Brahe et al [52] found that the US remission rates were only slightly lower and not statistically significant when using DAS28 over the other clinical criteria (SDAI, CDAI and ACR/EULAR). The authors also demonstrated that $1 / 3$ of GS synovial hypertrophy was detected in the feet. This finding may explain the lack of correlation between DAS28 remission and US remission [52].

The key strength of our study is that it was conducted in a real-life setting with a large cohort of RA patients, who were followed for a period of one year at five visits. The rate of achievement of clinical and imaging remission was compared separately for patients treated with sDMARDs and with bDMARDs. From our study, we draw 3 important conclusions. First, we ascertain that there is discordance between clinical remission by DAS28 and US remission as we found that imaging 
synovitis was present in patients with DAS28 remission. Second, we conclude that there is a higher rate of agreement between sonographic remission and clinical remission by the CDAI, SDAI and ACR/EULAR Boolean indices. Third, absence of GS imaging synovitis was not achieved within 12 months of therapy irrespective of the type of treatment.

There are also some limitations that need to be acknowledged. First, inter- and intra-observer agreement for the sonographic examination was not tested. The second and most important limitation refers to the lack of a gold standard for true remission against which the US method to be compared and evaluated as a biomarker of remission. The third limitation is the small number of patients fulfilling the stricter criteria for clinical remission (SDAI, CDAI, ACR/EULAR) which may have affected the precision of our estimates.

\section{Conclusions}

Clinical remission by DAS28 does not overlap imaging remission by MSUS. There is a persistence of imaging synovitis in RA patients in DAS28 clinical remission irrespective of the type of treatment. US may be a useful tool for the assessment of imaging remission and subclinical synovitis in patients in clinical remission.

\section{Conflict of interest: none}

\section{References}

1. Colebatch AN, Edwards CJ, Østergaard M, et al. EULAR recommendations for the use of imaging of the joints in the clinical management of rheumatoid arthritis. Ann Rheum Dis 2013;72:804-814.

2. Felson DT, Smolen JS, Wells G, et al. American College of Rheumatology/European League against Rheumatism provisional definition of remission in rheumatoid arthritis for clinical trials. Ann Rheum Dis 2011;70:404-413.

3. Molenaar ET, Voskuyl AE, Dinant HJ, Bezemer PD, Boers M, Dijkmans BA. Progression of radiologic damage in patients with rheumatoid arthritis in clinical remission. Arthritis Rheum 2004;50:36-42.

4. Brown AK, Conaghan PG, Karim Z, et al. An explanation for the apparent dissociation between clinical remission and continued structural deterioration in rheumatoid arthritis. Arthritis Rheum 2008;58:2958-2926.

5. Hammer HB, Kvien TK, Terslev L. Ultrasound of the hand is sufficient to detect subclinical inflammation in rheumatoid arthritis remission: a post hoc longitudinal study. Arthritis Res Ther 2017;19:221.

6. Gärtner M, Alasti F, Supp G, Mandl P, Smolen JS, Aletaha D. Persistence of subclinical sonographic joint activity in rheumatoid arthritis in sustained clinical remission. Ann Rheum Dis 2015;74:2050-2053

7. Naredo E, Bonilla G, Gamero F, Uson J, Carmona L, Laffon A. Assessment of inflammatory activity in rheumatoid arthritis: a comparative study of clinical evaluation with grey scale and power Doppler ultrasonography. Ann Rheum Dis 2005;64:375-381.

8. Vreju FA, Filippucci E, Gutierrez M, et al. Subclinical ultrasound synovitis in a particular joint is associated with ultrasound evidence of bone erosions in that same joint in rheumatoid patients in clinical remission. Clin Exp Rheumatol 2016;34:673-678.

9. Peluso G, Michelutti A, Bosello S, Gremese E, Tolusso B, Ferraccioli G. Clinical and ultrasonographic remission determines different chances of relapse in early and long standing rheumatoid arthritis. Ann Rheum Dis 2011;70:172-175.

10. Filippou G, Sakellariou G, Scirè CA, et al. The predictive role of ultrasound-detected tenosynovitis and joint synovitis for flare in patients with rheumatoid arthritis in stable remission. Results of an Italian multicentre study of the Italian Society for Rheumatology Group for Ultrasound: the STARTER study. Ann Rheum Dis 2018;77:1283-1289.

11. Prevoo ML, van 't Hof MA, Kuper HH, van Leeuwen MA, van de Putte LB, van Riel PL. Modified disease activity scores that include twenty-eight-joint counts. Development and validation in a prospective longitudinal study of patients with rheumatoid arthritis. Arthritis Rheum 1995;38:44-48.

12. Smolen JS, Breedveld FC, Schiff MH, et al. A simplified disease activity index for rheumatoid arthritis for use in clinical practice. Rheumatology (Oxford) 2003;42:244257.

13. Aletaha D, Nell VP, Stamm T, et al. Acute phase reactants add little to composite disease activity indices for rheumatoid arthritis: validation of a clinical activity score. Arthritis Res Ther 2005; 7:R796-R806.

14. Naredo E, Valor L, De la Torre I, et al. Predictive value of Doppler ultrasound-detected synovitis in relation to failed tapering of biologic therapy in patients with rheumatoid arthritis. Rheumatology (Oxford) 2015;54:1408-1414.

15. Alivernini S, Peluso G, Fedele AL, Tolusso B, Gremese E, Ferraccioli G. Tapering and discontinuation of TNF- $\alpha$ blockers without disease relapse using ultrasonography as a tool to identify patients with rheumatoid arthritis in clinical and histological remission. Arthritis Res Ther 2016;18:39.

16. Iwamoto T, Ikeda K, Hosokawa J, et al. Prediction of relapse after discontinuation of biologic agents by ultrasonographic assessment in patients with rheumatoid arthritis in clinical remission: high predictive values of total gray-scale and power Doppler scores that represent residual synovial inflammation before discontinuation. Arthritis Care Res (Hoboken) 2014;66:1576-1581.

17. Marks JL, Holroyd CR, Dimitrov BD, et al. Does combined clinical and ultrasound assessment allow selection of individuals with rheumatoid arthritis for sustained reduction of anti-tumor necrosis factor therapy? Arthritis Care Res (Hoboken) 2015;67:746-753. 
18. Ben Abdelghani K, Miladi S, Souabni L, et al. Role of ultrasound in assessing remission in rheumatoid arthritis. Diagn Interv Imaging 2015;96:3-10.

19. Hammer HB, Kvien TK. Comparisons of 7- to 78-joint ultrasonography scores: all different joint combinations show equal response to adalimumab treatment in patients with rheumatoid arthritis. Arthritis Res Ther 2011;13:R78.

20. Wakefield RJ, Balint PV, Szkudlarek M, et al. Musculoskeletal ultrasound including definitions for ultrasonographic pathology. J Rheum 2005;32:2485-2487.

21. Scheel AK, Hermann KG, Kahler E, et al. A novel ultrasonographic synovitis scoring system suitable for analyzing finger joint inflammation in rheumatoid arthritis. Arthritis Rheum 2005;52:733-743.

22. Vlad V, Berghea F, Libianu S, et al. Ultrasound in rheumatoid arthritis - volar versus dorsal synovitis evaluation and scoring. BMC Musculoskelet Disord 2011;12:124.

23. Backhaus M, Ohrndorf S, Kellner H, et al. Evaluation of a novel 7-joint ultrasound score in daily rheumatologic practice: a pilot project. Arthritis Rheum 2009;61:11941201.

24. Backhaus T, Ohrndorf S, Kellner H, et al. The US7 score is sensitive to change in a large cohort of patients with rheumatoid arthritis over 12 months of therapy. Ann Rheum Dis 2013;72:1163-1169.

25. Balsa A, de Miguel E, Castillo C, Peiteado D, Martín-Mola E. Superiority of SDAI over DAS-28 in assessment of remission in rheumatoid arthritis patients using power Doppler ultrasonography as a gold standard. Rheumatology (Oxford) 2010;49:683-690.

26. Dejaco C, Duftner C, Wipfler-Freissmuth E, Weiss H, Graninger WB, Schirmer M. Ultrasound-defined remission and active disease in rheumatoid arthritis: association with clinical and serologic parameters. Semin Arthritis Rheum 2012;41:761-767.

27. Haavardsholm EA, Aga AB, Olsen IC, et al. Ultrasound in management of rheumatoid arthritis: ARCTIC randomised controlled strategy trial. BMJ 2016;354:i4205.

28. Saleem B, Brown AK, Keen H, et al. Disease remission state in patients treated with the combination of tumor necrosis factor blockade and methotrexate or with diseasemodifying antirheumatic drugs: A clinical and imaging comparative study. Arthritis Rheum 2009;60:1915-1922.

29. van der Ven M, Kuijper TM, Gerards AH, et al. No clear association between ultrasound remission and health status in rheumatoid arthritis patients in clinical remission. Rheumatology (Oxford) 2017;56:1276-1281.

30. Horton SC, Tan AL, Freeston JE, Wakefield RJ, Buch MH, Emery P. Discordance between the predictors of clinical and imaging remission in patients with early rheumatoid arthritis in clinical practice: implications for the use of ultrasound within a treatment-to-target strategy. Rheumatology (Oxford) 2016;55:1177-1187.

31. Aletaha D, Neogi T, Silman AJ, et al. 2010 Rheumatoid arthritis classification criteria: an American College of Rheumatology/European League Against Rheumatism collaborative initiative. Arthritis Rheum 2010;62:2569-2581.
32. Szkudlarek M, Court-Payen M, Jacobsen S, Klarlund M, Thomsen HS, Østergaard M. Interobserver agreement in ultrasonography of the finger and toe joints in rheumatoid arthritis. Arthritis Rheum 2003;48:955-962.

33. Naredo E, D'Agostino MA, Wakefield RJ, et al. Reliability of a consensus-based ultrasound score for tenosynovitis in rheumatoid arthritis. Ann Rheum Dis 2013;72:1328-1334.

34. IBM Corp. Released 2016. IBM SPSS Statistics for Windows, Version 24.0. Armonk, NY: IBM Corp.

35. Smolen JS, Breedveld FC, Burmester GR, et al. Treating rheumatoid arthritis to target: 2014 update of the recommendations of an international task force. Ann Rheum Dis 2016;75:3-15.

36. Smolen JS, Landewé R, Bijlsma J, et al. EULAR recommendations for the management of rheumatoid arthritis with synthetic and biological disease-modifying antirheumatic drugs: 2016 update. Ann Rheum Dis 2017;76:960977.

37. Lillegraven S, Prince FH, Shadick NA, et al. Remission and radiographic outcome in rheumatoid arthritis: application of the 2011 ACR/EULAR remission criteria in an observational cohort. Ann Rheum Dis 2012;71:681-686.

38. Ramírez J, Narváez JA, Ruiz-Esquide V, et al. Clinical and sonographic biomarkers of structural damage progression in RA patients in clinical remission: A prospective study with 12 months follow-up. Semin Arthritis Rheum 2017;47:303-309.

39. Saleem B, Brown AK, Keen H, et al. Should imaging be a component of rheumatoid arthritis remission criteria? A comparison between traditional and modified composite remission scores and imaging assessments. Ann Rheum Dis 2011;70:792-798.

40. D'Agostino MA, Terslev L, Wakefield R, et al. Novel algorithms for the pragmatic use of ultrasound in the management of patients with rheumatoid arthritis: from diagnosis to remission. Ann Rheum Dis 2016;75:1902-1908.

41. Haavardsholm EA, Lie E, Lillegraven S. Should modern imaging be part of remission criteria in rheumatoid arthritis? Best Pract Res Clin Rheumatol 2012;26:767-785.

42. Zufferey P, Scherer A, Nissen MJ, et al. Can ultrasound be used to predict loss of remission in patients with RA in a real-life setting? A Multicenter Cohort Study. J Rheumatol 2018;45:887-894.

43. Kavanaugh A, Firestein G, Boyle D. The biomarkers in rheumatology - promises and pitfalls. Future Rheumatol 2008;3:303-305.

44. Vreju F, Ciurea M, Roşu A, Muşetescu A, Grecu D, Ciurea P. Power Doppler sonography, a non-invasive method of assessment of the synovial inflammation in patients with early rheumatoid arthritis. Rom J Morphol Embryol 2011;52:637-643.

45. Ng N, Kelly S, Humby F, et al. OP0054 Ultrasound synovitis reflects synovial inflammation at a histopathological level. Ann Rheum Dis 2014;73:81.

46. Sakellariou G, Scirè CA, Verstappen SM, Montecucco C, Caporali R. In patients with early rheumatoid arthritis, the new ACR/EULAR definition of remission identifies pa- 
tients with persistent absence of functional disability and suppression of ultrasonographic synovitis. Ann Rheum Dis 2013;72:245-249.

47. Sokka T, Hetland ML, Mäkinen H, et al. Remission and rheumatoid arthritis: data on patients receiving usual care in twenty-four countries. Arthritis Rheum 2008;58:2642-2651.

48. Naredo E, Valor L, De la Torre I, et al. Ultrasound joint inflammation in rheumatoid arthritis in clinical remission: how many and which joints should be assessed? Arthritis Care Res (Hoboken) 2013;65:512-517.

49. Olmez MO, Gunal EK, Ureyen SB, et al. Comparison of composite indices with global synovitis score on ultrasound for detecting remission. Clin Rheumatol 2018;37:1111-1114.
50. Witt M, Mueller F, Nigg A, et al. Relevance of grade 1 grayscale ultrasound findings in wrists and small joints to the assessment of subclinical synovitis in rheumatoid arthritis. Arthritis Rheum 2013;65:1694-1701.

51. Spinella A, Sandri G, Carpenito G, Belletti L, Mascia MT. The discrepancy between clinical and ultrasonographic remission in rheumatoid arthritis is not related to therapy or autoantibody status. Rheumatol Int 2012;32:3917-3921.

52. Brahe C, Terslev L, Krabbe S, et al. AB0263 Agreement between Das28, Acr/eular, Sdai, Cdai and ultrasound remission in patients with rheumatoid arthritis receiving biological treatment in routine care. Ann Rheum Dis 2016;75:989990. 